\title{
INFLUÊNCIA DA SATURAÇÃO POR BASES E DO FÓSFORO NO CRESCIMENTO DE MUDAS DE ANGICO-BRANCO ${ }^{1}$
}

\author{
Keli Cristina de Oliveira Gomes ${ }^{2}$, Haroldo Nogueira de Paiva ${ }^{3}$, Júlio César Lima Neves ${ }^{4}$, Nairam Félix de \\ Barros $^{4}$ e Sérgio Ricardo Silva ${ }^{5}$
}

\begin{abstract}
RESUMO - Este trabalho teve por objetivo avaliar o desempenho de mudas da espécie angico-branco (Anadenanthera colubrina (Vell.) Brenan), em condições diferenciadas de disponibilidade de fósforo no solo e de saturação por bases. Os tratamentos foram representados por um fatorial de seis níveis de $\mathrm{P}(0,100,200,300,400$ e $500 \mathrm{mg} / \mathrm{dm}^{3}$ ) por cinco níveis de saturação por bases (original =24, 40, 50,60 e 70\%), sendo dispostos no delineamento em blocos ao acaso, com cinco repetições. Aos 170 dias após a semeadura, foram feitas medições de altura e diâmetro do coleto de todas as plantas, as quais foram colhidas, separadas em raiz e parte aérea e postas a secar para obtenção do peso de matéria seca. A adição da mistura corretiva ao substrato não apresentou resposta significativa por parte da espécie estudada. Essa falta de resposta pode estar relacionada ao fato de que os teores de $\mathrm{Ca}\left(0,9 \mathrm{cmol}_{\mathrm{c}} / \mathrm{dm}^{3}\right)$ e de $\mathrm{Mg}\left(0,4 \mathrm{cmol} / \mathrm{dm}^{3}\right)$ contidos no solo utilizado tenham sido suficientes para suprir as necessidades das plantas nessa fase. No entanto, observou-se que o angico-branco responde positivamente à aplicação de P. Considerando-se a saturação por bases de 40 e $60 \%$, as doses de P que proporcionaram $90 \%$ de produção máxima, com base na produção de matéria seca da parte aérea, foram de 127 e $126 \mathrm{mg} / \mathrm{dm}^{3}$, respectivamente. De acordo com o modelo ajustado para $\mathrm{P}$ recuperado, em função do $\mathrm{P}$ adicionado, a dose que proporcionará essa disponibilidade de fósforo aos 170 dias é de $976,2 \mathrm{mg} / \mathrm{dm}^{3}$ para $\mathrm{V}=40 \%$ e de $1.277,3 \mathrm{mg} / \mathrm{dm}^{3}$ para $\mathrm{V}=60 \%$. O nível crítico de $\mathrm{P}$ no solo, pelo extrator Mehlich-1, foi de $13,88 \mathrm{mg} / \mathrm{dm}^{3}(\mathrm{~V}=40 \%)$ e de $12,87 \mathrm{mg} / \mathrm{dm}^{3}(\mathrm{~V}=60 \%)$ e na parte aérea, de $0,14 \mathrm{dag} / \mathrm{kg}(\mathrm{V}=40 \%)$ e de 0,12 $\mathrm{dag} / \mathrm{kg}(\mathrm{V}=60 \%)$.
\end{abstract}

Palavras-chave: Anadenanthera colubrina, angico-branco, saturação por bases e fósforo.

\section{INFLUENCE OF BASE SATURATION AND PHOSPHORUS ON THE DEVELOPMENT OF ANGICO-BRANCO SEEDLINGS}

\begin{abstract}
This study aimed at evaluating the seedling performance of angico-branco (Anadenanthera colubrina (Vell.) Brenan), under different conditions of phosphorus availability and variable levels of base saturation. The treatments were represented by a 6 (P doses: $0,100,200,300,400$ and $500 \mathrm{mg}^{2} \mathrm{dm}^{3}$ ) by 5 (percentage of basis saturation: original, 40, 50, 60 and 70\%) factorial arrangement, distributed in randomized blocks with five replications. 170 days after sowing, stalk height and diameter of all "angico-branco" plants were measured, finishing the experiment. The addition of corrective mixed to substrate did not show significant response on the species studied. This absence of response can be related to the fact that the levels of Ca $\left(0,9 \mathrm{cmol} / \mathrm{dm}^{3}\right)$ and $\mathrm{Mg}\left(0,4 \mathrm{cmol} / \mathrm{dm}^{3}\right)$ in the soil used, might be efficient to supply the needs of the plants
\end{abstract}

\footnotetext{
${ }^{1}$ Recebido em 2.04.2003 e aceito para publicação em 10.08.2004.

2 Engenheira Florestal. E-mail: <kelicog@bol.com.br>.

${ }^{3}$ Departamento de Engenharia Florestal da UFV, 36571-000 Viçosa, MG. E-mail: <hnpaiva@ mail.ufv.br>.

${ }^{4}$ Departamento de Solos da UFV, 36570-000 Viçosa - MG. E-mails:<julio@ solos.ufv.br $>$ e <nfbarros@ mail.ufv.br>.

${ }^{5}$ Programa de Pós-Graduação em Solos e Nutrição de Plantas da UFV. E-mail: <sergioufv@ yahoo.com.br>.
} 
in that phase. However, it was observed that "angico-branco" responded positively to P fertilization. P doses that leaded to $90 \%$ of the maximum dry matter production were $127 \mathrm{mg} / \mathrm{dm}^{3}$ on $40 \%$ base saturation and $126 \mathrm{mg} / \mathrm{dm}^{3}$ on $60 \%$ base saturation.According to the adjusted model for recorred $P$, relative to addedP; the dose that lead to available $P$ at todays were $976,2 \mathrm{mg} / \mathrm{dm}^{3}$, with $\mathrm{V}=40 \%$ and $1277,3 \mathrm{mg} / \mathrm{dm}^{3}$, with $\mathrm{V}=60 \%$. The critical $P$ level at $V=40 \%$ and $V=60 \%$ were 13,88 and $12,87 \mathrm{mg} / \mathrm{dm}^{3}$ in the soil and 0,14 and 0,12 dag/ $\mathrm{kg}$ on the shoot, respectively.

Kew words: Anadenanthera colubrina, "angico-branco", base saturation and phosphorus.

\section{INTRODUÇÃO}

Anadenanthera colubrina é uma espécie arbórea da família Leguminosae - Mimosoideae, com altura variando de 12 a $15 \mathrm{~m}$ e diâmetro entre 30 e $50 \mathrm{~cm}$. É particularmente freqüente nas regiões mais altas da encosta atlântica nos Estados do Rio de Janeiro e São Paulo. Produz anualmente grande quantidade de sementes viáveis (LORENZI, 1992).

Sua madeira apresenta grande durabilidade quando exposta, podendo ser utilizada em tacos, marcenaria, obras internas e construção civil e naval, dentre outros (CARVALHO, 1994). Essa espécie pode ser usada ainda no controle da erosão e no melhoramento de solos (SANTOS, 1987). Segundo Lorenzi (1992), a espécie pode ser aproveitada para a arborização de parques e praças e para o plantio em florestas mistas destinadas à recomposição de áreas degradadas de preservação permanente.

Algumas espécies nativas possuem características interessantes, por isso merecem maiores estudos (DIAS et al., 1991). Devido ao crescente processo de devastação das áreas de nascentes e das matas ciliares, juntamente com a necessidade de reflorestamento em solos com características químicas diferentes, o conhecimento sobre as demandas nutricionais das espécies utilizadas para essas finalidades, entre outras, é de fundamental importância (FERNANDES et al., 2000; FURTINI NETO et al., 2000).

Segundo Parrota (1992), citado por Faria et al. (1995), os programas de revegetação têm buscado explorar o potencial de espécies nativas, por estas se adaptarem melhor às condições edafoclimáticas e facilitarem o restabelecimento do equilíbrio entre a fauna e a flora. Outro aspecto a ser considerado refere-se à importância das florestas nativas no contexto da produção de madeira e da conservação ambiental (DUBOC et al., 1996). Logo, pesquisas que visem avaliar o comportamento de espécies florestais que possam apresentar bom desempenho e que possuam características interessantes a diversas finalidades de uso são de grande valia. Nesse sentido, trabalhos como o de Meira Neto (1997), que realizou as caracterizações florística, estrutural e ambiental de estratos arbóreos e herbáceo-arbustivo de uma Floresta Estacional Semidecidual em Viçosa podem embasar, em parte, estudos dessa natureza.

É sabido que o fósforo é um dos elementos mais limitantes à produção das culturas em condições de solos tropicais, devido à característica de "solo-dreno" que estes apresentam (NOVAIS e SMYTH, 1999). A maioria das terras destinadas ao reflorestamento apresenta baixa disponibilidade de fósforo. Além disso, para a produção de mudas é comum o uso de substratos compostos de solos advindos dessas mesmas áreas. Assim, este elemento se torna restritivo à produção de mudas de boa qualidade (NOVAIS et al., 1990).

Este trabalho foi realizado com o objetivo de avaliar a influência de níveis de saturação por bases e doses de fósforo sobre o crescimento da espécie angico-branco (Anadenanthera colubrina (Vell.) Brenan), uma vez que ela foi listada como mais importante (IVI), em pelo menos três matas mesófilas estudadas fitossociologicamente em Minas Gerais (MEIRA NETO et al., 1997).

\section{MATERIAL E MÉTODOS}

Este trabalho foi desenvolvido no Viveiro de Pesquisa do Departamento de Engenharia Florestal, pertencente à Universidade Federal de Viçosa, em Viçosa, MG.

Os tratamentos foram representados por um fatorial de seis níveis de $\mathrm{P}\left(0,100,200,300,400\right.$ e $\left.500 \mathrm{mg} / \mathrm{dm}^{3}\right)$ 
por cinco níveis de saturação por bases (original $=24$, 40, 50, 60 e 70\%), sendo dispostos no delineamento em blocos ao acaso, com cinco repetições. A parcela experimental foi constituída por um vaso de polietileno rígido, contendo cada um $2 \mathrm{~kg}$ de solo. A espécie utilizada foi angico-branco (Anadenanthera colubrina (Vell.) Brenan).

Como substrato, utilizou-se uma amostra retirada da camada de $0-20 \mathrm{~cm}$ de profundidade de um Latossolo Vermelho Distrófico, textura argilosa, retirado de uma área próxima à região de Viçosa, MG, sendo posteriormente secado ao ar, peneirado (malha de $4 \mathrm{~mm}$ de diâmetro) e destinado ao enchimento dos vasos. Desse solo, coletou-se uma amostra que foi caracterizada física ( $\operatorname{argila}=450 \mathrm{~g} / \mathrm{kg}$, silte $=90 \mathrm{~g} /$ $\mathrm{kg}$, areia grossa $=210 \mathrm{~g} / \mathrm{kg}$ e areia fina $=250 \mathrm{~g} / \mathrm{kg}$ ) e quimicamente (Quadro 1). A seguir, o solo recebeu cinco níveis de calagem, de acordo com o método de elevação da saturação por bases, ou seja, 24\% (saturação por bases em condições naturais), e elevação da saturação por bases para 40, 50, 60 e 70\%, utilizando-se da seguinte fórmula para cálculo:

$\mathrm{NC}(\mathrm{t} / \mathrm{ha})=\left(\mathrm{V}_{2}-\mathrm{V}_{1}\right) \mathrm{T} / 100$

Quadro 1-Características químicas da amostra de solo utilizado para a produção de mudas de Anadenanthera colubrina (Vell.) Brenan (angico-branco)

Table 1 - Chemical characteristics of soil sample used to produce seedlings of Anadenanthera colubrina (Vell.) Brenan (angico-branco)

\begin{tabular}{lc}
\hline Característica & Valor \\
\hline $\mathrm{pH}{ }^{1}$ & 5,1 \\
$\mathrm{P}\left(\mathrm{mg} / \mathrm{dm}^{3}\right)^{2}$ & 4,8 \\
$\mathrm{~K}\left(\mathrm{mg} / \mathrm{dm}^{3}\right)^{2}$ & 68 \\
$\mathrm{Al}{ }^{3+}\left(\mathrm{cmol}_{\mathrm{c}} / \mathrm{dm}^{3}\right)^{3}$ & 0,1 \\
$\mathrm{Ca}^{2+}\left(\mathrm{cmol}_{\mathrm{c}} / \mathrm{dm}^{3}\right)^{3}$ & 0,9 \\
$\mathrm{Mg}{ }^{2+}\left(\mathrm{cmol}_{\mathrm{c}} / \mathrm{dm}^{3}\right)^{3}$ & 0,4 \\
$\mathrm{H}+\mathrm{Al}\left(\mathrm{cmol}_{\mathrm{c}} / \mathrm{dm}^{3}\right)^{3}$ & 4,6 \\
$\mathrm{SB}\left(\mathrm{cmol} / \mathrm{dm}^{3}\right)^{5}$ & 1,47 \\
$\mathrm{CTC}(\mathrm{t})\left(\mathrm{cmol}_{\mathrm{c}} / \mathrm{dm}^{3}\right)^{6}$ & 1,57 \\
$\mathrm{CTC}(\mathrm{T})\left(\mathrm{cmol}_{\mathrm{c}} / \mathrm{dm}^{3}\right)^{7}$ & 6,07 \\
$\mathrm{~V}(\%)^{8}$ & 24 \\
$\mathrm{MO}(\mathrm{dag} / \mathrm{kg})^{9}$ & 2,46 \\
\hline
\end{tabular}

${ }^{1} \mathrm{pH}$ em $\mathrm{H}_{2} \mathrm{O} 1: 2,5$.

${ }^{2}$ Extrator Mehlich-1.

${ }^{3}$ Extrator $\mathrm{KCl} 1,0 \mathrm{~mol} / \mathrm{L}$

${ }^{4}$ Extrator $\mathrm{CaOAc} 0,5 \mathrm{~mol} / \mathrm{L}, \mathrm{pH} 7,0$.

${ }^{5} \mathrm{SB}=\mathrm{K}^{+}+\mathrm{Ca}^{2+}+\mathrm{Mg}^{2+}$

${ }^{6}$ Capacidade de troca catiônica efetiva.

${ }^{7}$ Capacidade de troca catiônica a pH 7,0.

${ }^{8}$ Índice de saturação por bases.

${ }^{9}$ Matéria orgânica (MO=C. org. x 1,724-Walkley-Black).
Em que:

$\mathrm{NC}=$ necessidade de calagem (toneladas/hectare);

V2 = porcentagem de saturação por bases desejada;

V1 = porcentagem de saturação por bases do solo, conforme análise;

$\mathrm{T}=\mathrm{CTC}$ a pH 7,0.

$\mathrm{O}$ corretivo consistiu numa mistura de $\mathrm{CaCO}_{3} \mathrm{e}$ $\mathrm{MgCO}_{3}$, na relação estequiométrica de 4:1. Após a aplicação do corretivo, seguiu-se um período de incubação de 30 dias, sendo o teor de umidade mantido próximo à capacidade de campo, inclusive na porção que não recebeu calcário.

As fontes de $\mathrm{P}$ utilizadas foram $\mathrm{NaH}_{2} \mathrm{PO}_{4} \cdot \mathrm{H}_{2} \mathrm{O}$ e $\mathrm{KH}_{2} \mathrm{PO}_{4}$. Juntamente com o fósforo, realizou-se uma adubação básica, aplicada dois dias antes da semeadura, que consistiu na aplicação, por solução, de 100 $\mathrm{mg} / \mathrm{dm}^{3}$ de $\mathrm{N}, 100 \mathrm{mg} / \mathrm{dm}^{3}$ de $\mathrm{Ke} 40 \mathrm{mg} / \mathrm{dm}^{3}$ de S, pelo emprego de $\mathrm{NH}_{4} \mathrm{NO}_{3}, \mathrm{KCl}$ e $\mathrm{K}_{2} \mathrm{SO}_{4}$, conforme sugerido por Passos (1994). Recebeu ainda uma solução de micronutrientes, nas seguintes doses: $\mathrm{B}=0,81 \mathrm{mg} / \mathrm{dm}^{3}$ $\left(\mathrm{H}_{3} \mathrm{BO}_{3}\right), \mathrm{Mn}=3,66 \mathrm{mg} / \mathrm{dm}^{3}\left(\mathrm{MnCl}_{2} \cdot \mathrm{H}_{2} \mathrm{O}\right), \mathrm{Zn}=4,00 \mathrm{mg} /$ $\mathrm{dm}^{3}\left(\mathrm{ZnSO}_{4} \cdot 7 \mathrm{H}_{2} \mathrm{O}\right), \mathrm{Cu}=1,33 \mathrm{mg} / \mathrm{dm}^{3}\left(\mathrm{CuSO}_{4} \cdot 5 \mathrm{H}_{2} \mathrm{O}\right)$ e $\mathrm{Mo}=0,15 \mathrm{mg} / \mathrm{dm}^{3}\left(\left(\mathrm{NH}_{4}\right)_{6} \mathrm{Mo}_{7} \mathrm{O}_{24} \cdot 4 \mathrm{H}_{2} \mathrm{O}\right)$, de acordo com Alvarez V. (1974). Após a aplicação, procedeu-se à mistura das soluções com o solo das unidades experimentais.

Foram realizadas, também, mais três adubações nitrogenadas, aos 35, 70 e 105 dias após a semeadu$\mathrm{ra}$, na dose de $20 \mathrm{mg} / \mathrm{dm}^{3}$ de N, por aplicação, utilizando-se como fontes do elemento, nas duas primeiras épocas, o $\mathrm{NH}_{4} \mathrm{NO}_{3} \mathrm{e}$, na última, o $\mathrm{KNO}_{3}$. Nessa última aplicação, juntamente com o $\mathrm{N}$, foram adicionados 55,8 $\mathrm{mg} / \mathrm{dm}^{3}$ de K, conforme sugerido por Garcia (1986).

Cada vaso plástico recebeu cinco sementes, efetuando-se o primeiro desbaste aos 15 dias após a emergência, deixando-se duas plantas por vaso. Após 30 dias, um segundo desbaste foi realizado, deixando-se apenas uma muda por vaso.

Durante o período experimental, a umidade do solo foi mantida próxima de $60 \%$ da capacidade de campo. A irrigação foi feita utilizando água deionizada, procedendo-se ao monitoramento diário para esse controle.

R. Árvore, Viçosa-MG, v.28, n.6, p.785-792, 2004 
Aos 170 dias após a semeadura, foram medidos a altura e o diâmetro do coleto de todas as plantas. A altura foi medida utilizando régua graduada em centímetros e a medição do diâmetro do coleto, realizada por meio de um paquímetro digital, com precisão de $0,01 \mathrm{~mm}$. As plantas foram separadas em raiz e parte aérea, lavadas com água destilada e secadas em estufa com circulação forçada de ar, a uma temperatura próxima a $70^{\circ} \mathrm{C}$, até atingir peso constante. Foram, então, pesadas, em balança analítica com precisão de $0,01 \mathrm{~g}$ e moídas em moinho tipo Willey, com peneira de $2 \mathrm{~mm}$ de abertura de malha. O material vegetal secado e moído foi mineralizado, pela mistura nítrico-perclórica, e nos extratos se procedeu à determinação de $\mathrm{P}$. $\mathrm{O}$ fósforo foi dosado por colorimetria pelo método da vitamina $\mathrm{C}$ (BRAGA e DEFELIPO, 1974) em espectrofotômetro a $725 \mathrm{~nm}$.

Ao final da experimentação, o solo contido nos vasos foi secado ao ar e peneirado (malha de $4 \mathrm{~mm}$ de diâmetro), sendo, então, destinado à análise de $\mathrm{P}$ disponível, pelo Mehlich -1 (EMBRAPA, 1997).

Foram ajustadas equações de regressão, dos valores de altura, diâmetro do coleto, peso de matéria seca e teores de $\mathrm{P}$ na parte aérea das plantas em função das doses de $\mathrm{P}$ aplicadas, dentro de cada nível de saturação por bases. As doses recomendáveis de $\mathrm{P}$ foram aquelas responsáveis pela obtenção de $90 \%$ da produção máxima estimada de matéria seca da parte aérea. Substituindo essas doses nas equações obtidas para o $\mathrm{P}$ recuperado, pelo Mehlich-1, em função do $\mathrm{P}$ adicionado, foram obtidos os níveis críticos de $\mathrm{P}$ no solo. Já, na parte aérea, os níveis críticos de $\mathrm{P}$ foram resultantes da substituição das doses recomendáveis nas equações relacionando os teores de P nessa parte das plantas em função das doses adicionadas.

\section{RESULTADOS E DISCUSSÃO}

A adição da mistura corretiva ao substrato não resultou em resposta significativa por parte das plantas de angico-branco. O mesmo comportamento tem sido observado nas diferentes espécies florestais com relação à calagem (DIAS et al., 1990, 1991; BALIERO et al., 1995). Essa falta de resposta pode estar relacionada ao fato de que os teores de $\mathrm{Ca}\left(0,9 \mathrm{cmol}_{\mathrm{c}} / \mathrm{dm}^{3}\right)$ e de $\mathrm{Mg}\left(0,4 \mathrm{cmol} / \mathrm{dm}^{3}\right)$ contidos no solo utilizado, tenham sido suficientes para suprir as necessidades das plantas nessa fase. No entanto, observou-se que o angicobranco responde positivamente à aplicação de $\mathrm{P}$, apresentando, na fase inicial de crescimento, uma demanda relativamente elevada de fósforo, sendo a disponibilidade original do solo estudado e também a dos solos da Zona da Mata mineira, insuficiente para suprir as exigências nutricionais desta espécie, nesta fase.

Em alguns níveis de saturação por bases (V\%), a altura, o diâmetro do coleto e o peso de matéria seca da parte aérea das plantas, aos 170 dias após a semeadura, aumentaram com a elevação da dose de $\mathrm{P}$ adicionada ao solo. De maneira geral, o aumento nas doses de $\mathrm{P}$ aplicadas também possibilitou incremento nos teores desse nutriente na parte aérea (Quadro 2). No entanto, nem sempre o aumento do teor foliar reflete na produção de matéria seca, e a faixa em que isso ocorre é identificada como zona de alimentação de luxo (MALAVOLTA et al., 1997).

Devido à alta correlação entre a matéria seca da parte aérea e às características de crescimento, altura e diâmetro do coleto, as tendências em relação à aplicação de $\mathrm{P}$ foram bastante semelhantes. Para o crescimento em altura, tal tendência seguiu uma variação quadrática para $V=40$ e $60 \%$ de saturação por bases; nos demais níveis, não houve resposta significativa à aplicação de P. Para o diâmetro do coleto houve resposta (quadrática) à aplicação de $\mathrm{P}$ apenas no nível de $40 \%$ de saturação por bases. Para a matéria seca da parte aérea, ajustaram-se modelos quadráticos para $V=40$ e $60 \%$. Nos demais níveis de saturação por bases não houve resposta significativa à aplicação de $\mathrm{P}$ (Quadro 3 ).

O comportamento quadrático do crescimento de mudas de espécies nativas, em resposta à aplicação de P, também foi observado em Sclerolobium paniculatum Vogel (táxi-branco) (DIAS et al.,1991), Cedrela fissilis Vell. (cedro) (GARCIA, 1986), Chorisia speciosa A.St.-Hil. (paineira) (FERNANDES et al., 2000) e, ainda, para, Dalbergia nigra Willd. (Jacarandá-daBahia) (REIS et al., 1997).

Os modelos ajustados (Quadro 4) permitiram a obtenção de níveis críticos de $\mathrm{P}$ no solo e na matéria seca da parte aérea (Quadro 5). No angico-branco, a dose de $\mathrm{P}$ que proporcionou $90 \%$ de produção máxima, com base na produção de matéria seca da parte aérea, foi igual a $127,0 \mathrm{mg} / \mathrm{dm}^{3}$ no nível de saturação por bases de $40 \%$ e $126,0 \mathrm{mg} / \mathrm{dm}^{3}$ no nível de $60 \%$. De acordo com o modelo ajustado para $\mathrm{P}$ recuperado, em função do $\mathrm{P}$ adicionado, a dose que proporcionará essa disponibilidade de fósforo aos 170 dias será de $976,2 \mathrm{mg} / \mathrm{dm}^{3}$ para $\mathrm{V}=40 \%$ e de $1.277,3 \mathrm{mg} / \mathrm{dm}^{3}$ para $\mathrm{V}=60 \%$. 
Quadro 2- Médias das seguintes características do angico-branco: altura (Alt), diâmetro do coleto (Diam), peso de matéria seca da parte aérea (Mspa), peso de matéria seca de raiz (Msra), relação raiz/parte aérea (Rrpa) e teor de P na matéria seca da parte aérea (TPpa) aos 170 dias após a semeadura

Table 2 -Average for angico-branco's characteristics: height (Alt), stem diameter (Diam), aerial part dry matter (Mspa), root dry matter (Msra), ratio of root biomass/aerial biomass (Rrpa) and P concentrations in shoot dry matter 170 days after sowing

\begin{tabular}{|c|c|c|c|c|c|c|c|}
\hline $\begin{array}{c}\text { Saturação por } \\
\text { bases }(\%)\end{array}$ & $\begin{array}{c}\text { Dose P } \\
\left(\mathrm{mg} / \mathrm{dm}^{3}\right)\end{array}$ & $\begin{array}{c}\text { Alt } \\
(\mathrm{cm})\end{array}$ & $\begin{array}{l}\text { Diam. } \\
(\mathrm{mm})\end{array}$ & $\begin{array}{c}\text { Mspa } \\
\text { (g/vaso) }\end{array}$ & $\begin{array}{c}\text { Msra } \\
\text { (g/vaso) }\end{array}$ & $\begin{array}{l}\text { Rrpa } \\
(\mathrm{g} / \mathrm{g})\end{array}$ & $\begin{array}{c}\text { TPpa } \\
(\mathrm{dag} / \mathrm{kg})\end{array}$ \\
\hline 24 & 0 & 20,50 & 4,13 & 2,75 & 0,84 & 0,30 & 0,073 \\
\hline 24 & 100 & 24,86 & 6,05 & 4,73 & 1,78 & 0,40 & 0,132 \\
\hline 24 & 200 & 24,74 & 4,53 & 3,26 & 1,04 & 0,30 & 0.135 \\
\hline 24 & 300 & 22,72 & 4,61 & 3,03 & 0,91 & 0,29 & 0,204 \\
\hline 24 & 400 & 27,10 & 5,77 & 4,65 & 1,41 & 0,29 & 0,268 \\
\hline 24 & 500 & 26,94 & 5,16 & 4,15 & 1,07 & 0,24 & 0,337 \\
\hline 40 & 0 & 19,74 & 4,28 & 2,62 & 0,87 & 0,37 & 0,082 \\
\hline 40 & 100 & 21,40 & 5,26 & 3,82 & 1,70 & 0,41 & 0,127 \\
\hline 40 & 200 & 26,92 & 5,80 & 4,64 & 1,70 & 0,37 & 0,184 \\
\hline 40 & 300 & 33,38 & 5,26 & 4,98 & 1,49 & 0,30 & 0,195 \\
\hline 40 & 400 & 25,14 & 4,12 & 3,68 & 0,79 & 0,22 & 0,183 \\
\hline 40 & 500 & 10,60 & 2,33 & 0,77 & 0,12 & 0,14 & 0,230 \\
\hline 50 & 0 & 19,30 & 4,50 & 3,57 & 1,27 & 0,35 & 0,077 \\
\hline 50 & 100 & 22,30 & 6,38 & 5,32 & 2,04 & 0,39 & 0,129 \\
\hline 50 & 200 & 32,78 & 6,13 & 5,17 & 1,86 & 0,36 & 0,184 \\
\hline 50 & 300 & 17,78 & 4,76 & 3,95 & 1,36 & 0,33 & 0,213 \\
\hline 50 & 400 & 22,18 & 4,11 & 3,29 & 0,73 & 0,22 & 0,233 \\
\hline 50 & 500 & 21,82 & 4,67 & 4,26 & 1,08 & 0,25 & 0,494 \\
\hline 60 & 0 & 15,02 & 4,14 & 2,89 & 1,17 & 0,37 & 0,095 \\
\hline 60 & 100 & 21,22 & 4,30 & 3,73 & 1,12 & 0,29 & 0,135 \\
\hline 60 & 200 & 27,60 & 4,72 & 3,90 & 1,17 & 0,27 & 0,137 \\
\hline 60 & 300 & 28,88 & 4,87 & 4,65 & 1,29 & 0,26 & 0,196 \\
\hline 60 & 400 & 19,74 & 4,59 & 4,09 & 1,04 & 0,23 & 0,198 \\
\hline 60 & 500 & 21,50 & 4,06 & 2,96 & 0,72 & 0,23 & 0,308 \\
\hline 70 & 0 & 18,88 & 4,34 & 2,96 & 1,11 & 0,37 & 0,078 \\
\hline 70 & 100 & 29,38 & 4,74 & 3,84 & 1,47 & 0,31 & 0,111 \\
\hline 70 & 200 & 25,12 & 4,46 & 3,29 & 0,95 & 0,26 & 0,114 \\
\hline 70 & 300 & 20,12 & 4,23 & 3,36 & 0,95 & 0,27 & 0,199 \\
\hline 70 & 400 & 31,40 & 4,16 & 3,77 & 0,75 & 0,18 & 0,222 \\
\hline 70 & 500 & 18,25 & 4,32 & 3,06 & 0,60 & 0,19 & 0,325 \\
\hline
\end{tabular}

Com relação à produção de matéria seca de raiz, não houve resposta significativa nos níveis de saturação por bases de 24 e $60 \%$. O maior valor estimado (1,91 g/vaso) foi obtido com a dose de aproximadamente $5 \mathrm{mg} / \mathrm{dm}^{3}$ de $\mathrm{P}$, no nível de saturação por bases igual a $50 \%$. Para $\mathrm{V}=70 \%$, a produção de matéria seca de raiz seguiu uma variação linear negativa em relação às doses de $\mathrm{P}$ (Quadro 6). A queda na produção de biomassa de raiz, no mais alto nível de adubação, pode estar relacionada à maior alocação de nutrientes para produção de biomassa da parte aérea (FURTINI NETO, 1994). Esse mesmo comportamento foi observado na aroeirinha, paineira e jambolão, ou seja, com o aumento do fornecimento de $\mathrm{P}$ ocorreu sensível incremento na produção de matéria seca da parte aérea em relação à matéria seca de raiz (FERNANDES et al., 2000).

A relação raiz/parte aérea apresentou comportamento bem distinto nos diferentes níveis de saturação por bases (Quadro 6), variando entre modelos cúbico raiz ( $\mathrm{V}=24 \%)$, raiz quadrático $(40 \%)$ e linear negativo ( $\mathrm{V}=50,60$ e $70 \%)$, sendo os maiores valores médios obtidos entre as doses de 0 e $100 \mathrm{mg} / \mathrm{dm}^{3}$ de P. Segundo Glass (1989), a razão entre a biomassa de raízes e a da parte aérea aumenta à medida que diminui o suprimento de nutrientes. Portanto, a sua diminuição está relacionada com uma melhor nutrição de fósforo. O maior valor estimado $(0,43 \mathrm{~g} / \mathrm{g})$ foi atingido no

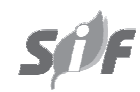

R. Árvore, Viçosa-MG, v.28, n.6, p.785-792, 2004 
nível de $40 \%$ de saturação por bases, com a dose de $45 \mathrm{mg} / \mathrm{dm}^{3}$ de P. Esse valor da razão biomassa de raiz/ biomassa da parte aérea é semelhante ao encontrado em Acacia mangium (0,50 g/g) (DANIEL et al., 1997).
Segundo esses mesmos autores, a escolha da dose de fósforo a ser aplicada depende das necessidades na produção de mudas, ou seja, da característica que é mais desejável.

Quadro 3 - Equações de regressão da altura, diâmetro do coleto, matéria seca da parte aérea e teor de P na parte aérea, como variáveis dependentes das doses de $\mathrm{P}$ nos diferentes níveis de saturação por bases, para produção de mudas de angico-branco (Anadenanthera colubrina)

Table 3 - Regression equations for height, stem diameter, dry matter productions of the aerial part and P concentrations in shoot dry matter as dependent variables of $P$ doses in different base saturation for angico-branco (Anadenanthera colubrina) seedling production

\begin{tabular}{|c|c|c|c|}
\hline Variável & $\begin{array}{c}\text { Saturação por } \\
\text { Bases }\end{array}$ & Equação & $\mathrm{R}^{2}$ \\
\hline \multirow[t]{5}{*}{ Altura } & 24 & $\hat{\mathrm{Y}}=\mathrm{Y}=24,48$ & \\
\hline & 40 & $\hat{Y}=16,767+0,1135 * * X-0,0002499 * * X^{2}$ & 0,7861 \\
\hline & 50 & $\hat{\mathrm{Y}}=\mathrm{Y}=22,69$ & \\
\hline & 60 & $\hat{Y}=15,221+0,08360 * X-0,0001505 * X^{2}$ & 0,7291 \\
\hline & 70 & $\hat{\mathrm{Y}}=\mathrm{Y}=20,03$ & \\
\hline \multirow{5}{*}{$\begin{array}{l}\text { Diâmetro do } \\
\text { coleto }\end{array}$} & 24 & $\hat{\mathrm{Y}}=\mathrm{Y}=5,04$ & \\
\hline & 40 & $\hat{\mathrm{Y}}=4,266+0,1445 * * \mathrm{X}-0,0003677 * \mathrm{X}^{2}$ & 0,9969 \\
\hline & 50 & $\hat{\mathrm{Y}}=\mathrm{Y}=5,09$ & \\
\hline & 60 & $\hat{Y}=Y=4,45$ & \\
\hline & 70 & $\hat{\mathrm{Y}}=\mathrm{Y}=4,33$ & \\
\hline \multirow{5}{*}{$\begin{array}{l}\text { Matéria seca da } \\
\text { parte aérea }\end{array}$} & 24 & $\hat{Y}=Y=3,76$ & \\
\hline & 40 & $\hat{\mathrm{Y}}=2,355+0,02326 * * \mathrm{X}-0,00005182 * * \mathrm{X}^{2}$ & 0,9544 \\
\hline & 50 & $\hat{\mathrm{Y}}=\mathrm{Y}=3,49$ & \\
\hline & 60 & $\hat{\mathrm{Y}}=2,786+0,01206^{\circ} \mathrm{X}-0,00002285^{\circ} \mathrm{X}^{2}$ & 0,8754 \\
\hline & 70 & $\hat{\mathrm{Y}}=\mathrm{Y}=3,38$ & \\
\hline \multirow{5}{*}{$\begin{array}{l}\text { Teor de P na } \\
\text { matéria seca da } \\
\text { parte aérea }\end{array}$} & 24 & $\hat{\mathrm{Y}}=0,0632+0,0005126 * * \mathrm{X}$ & 0,9612 \\
\hline & 40 & $\hat{\mathrm{Y}}=0,0852+0,0004993^{*} * \mathrm{X}-0,0000004738^{14,7} \mathrm{X}^{2}$ & 0,9124 \\
\hline & 50 & $\hat{\mathrm{Y}}=0,084611618 \cdot(1,00320676 * *)^{\mathrm{x}}$ & 0,9282 \\
\hline & 60 & $\hat{Y}=0,105+0,00006292^{30,6} X+0,0000006251 * X^{2}$ & 0,9308 \\
\hline & 70 & $\hat{Y}=0,0826-0,007118 * * X^{0,5}+0,0007675 * * X$ & 0,9557 \\
\hline
\end{tabular}

**; $* \mathrm{e}^{\circ}$ coeficiente significativos a 1,5 e $10 \%$ de probabilidade, respectivamente.

Quadro 4 - Equações de regressão ajustadas da produção de matéria seca da parte aérea (Mspa), teor de P disponível no solo pelo extrator Mehlich-1 (P-disp.) e teor de $\mathrm{P}$ na matéria seca da parte aérea (P-ms), em função das doses de $\mathrm{P}$ aplicadas ao substrato

Table 4-Regression equations adjusted to dry matter productions of the aerial part (Mspa), soil concentrations of available $P$ Mehlich-1 extract ( $P$-disp) and $P$ concentrations in shoot dry matter $(P-m s)$, in function of $P$ doses applied to the substratum

\begin{tabular}{cclr}
\hline $\begin{array}{c}\text { Saturação por } \\
\text { Bases (\%) }\end{array}$ & Variável & Equação & $\mathrm{R}^{2}$ \\
\hline 40 & Mspa & $\hat{\mathrm{Y}}=2,355+0,02326^{* *} \mathrm{X}-0,00005182^{* *} \mathrm{X}^{2}$ & 0,95 \\
& P-disp. & $\hat{\mathrm{Y}}=-3,032+0,1332^{* *} \mathrm{X}$ & 0,96 \\
& P-ms & $\hat{\mathrm{Y}}=0,0852+0,0004993^{* *} \mathrm{X}-0,0000004738^{14,7} \mathrm{X}^{2}$ & 0,91 \\
60 & Mspa & $\hat{\mathrm{Y}}=2,786+0,01206^{\circ} \mathrm{X}-0,00002285^{\circ} \mathrm{X}^{2}$ & 0,87 \\
& P-disp. & $\hat{\mathrm{Y}}=0,484+0,09827 * \mathrm{X}$ & 0,98 \\
& P-ms & $\hat{\mathrm{Y}}=0,105+0,00006292^{30,6} \mathrm{X}+0,0000006251^{*} \mathrm{X}^{2}$ & 0,93 \\
\hline
\end{tabular}

$* * ; \mathrm{e}^{\circ}$ coeficiente significativo a 1,5 e $10 \%$ de probabilidade, respectivamente.

R. Árvore, Viçosa-MG, v.28, n.6, p.785-792, 2004 
Quadro 5 - Doses de fósforo recomendadas, níveis críticos no solo e na parte aérea das plantas de angico-branco, nos diferentes níveis de saturação por bases

Table 5-Recommended P doses, critical level of soil and aerial part of plant angico-branco, in the different base saturation

\begin{tabular}{cccc}
\hline Saturação por Bases & Dose de P recomendada & \multicolumn{2}{c}{ Nível Crítico de P } \\
\hline$(\%)$ & $\left(\mathrm{mg} / \mathrm{dm}^{3}\right)$ & No solo $\left(\mathrm{mg} / \mathrm{dm}^{3}\right)$ & Na parte aérea $(\mathrm{dag} / \mathrm{kg})$ \\
40 & 127 & 13,88 & 0,14 \\
60 & 126 & 12,87 & 0,12 \\
Média & 126,5 & 13,38 & 0,13 \\
\hline
\end{tabular}

Quadro 6 - Equações de regressão da produção de matéria seca de raiz e relação raiz/parte aérea, como variáveis dependentes das doses de P nos diferentes níveis de saturação por bases, para produção de mudas de angico-branco (Anadenanthera colubrina)

Table 6 - Regression equations for dry matter of the root and ratio of root biomass/aerial biomass as dependent variables of $P$ doses in the different base saturation for angico-branco (Anadenanthera colubrina) seedling production

\begin{tabular}{|c|c|c|c|}
\hline Variável & $\begin{array}{c}\text { Saturação por } \\
\text { Bases }\end{array}$ & Equação & $\mathbf{R}^{2}$ \\
\hline \multirow{5}{*}{$\begin{array}{l}\text { Matéria seca } \\
\text { de raiz }\end{array}$} & 24 & $\hat{\mathrm{Y}}=\mathrm{Y}=1,18$ & \\
\hline & 40 & $\hat{\mathrm{Y}}=0,973+0,007279 * * \mathrm{X}-0,00001836 * * \mathrm{X}^{2}$ & 0,9598 \\
\hline & 50 & $\hat{Y}=1,31696+0,126386^{* *} X^{0,5}-0,00676793^{* *} X$ & 0,7346 \\
\hline & 60 & $\hat{\mathrm{Y}}=\mathrm{Y}=1,09$ & \\
\hline & 70 & $\hat{\mathrm{Y}}=1,310-0,001351 * \mathrm{X}$ & 0,6976 \\
\hline \multirow{5}{*}{$\begin{array}{l}\text { Relação raiz/ } \\
\text { parte aérea }\end{array}$} & 24 & $\hat{Y}=0,300+0,003645^{\circ} X^{0,5}-0,003679^{13,0} X+0,00008807^{20,5} X^{1,5}$ & 0,8086 \\
\hline & 40 & $\hat{Y}=0,374+0,01592 * * X^{0,5}-0,001181 * * X$ & 0,9985 \\
\hline & 50 & $\hat{\mathrm{Y}}=0,391861-0,00029314 * * \mathrm{X}$ & 0,6975 \\
\hline & 60 & $\hat{\mathrm{Y}}=0,337-0,002464 * * \mathrm{X}$ & 0,7954 \\
\hline & 70 & $\hat{Y}=0,357-0,0003726 * * X$ & 0,9086 \\
\hline
\end{tabular}

**; $\mathrm{e}^{\circ}$ coeficiente significativos a 1,5 e $10 \%$ de probabilidade, respectivamente.

\section{CONCLUSÕES}

Os resultados permitiram a conclusão de que:

- A adição da mistura corretiva ao substrato e o aumento da saturação de bases não apresentaram resposta significativa por parte da espécie estudada.

- A resposta à adubação fosfatada pelo angicobranco foi quadrática, para a saturação de bases de 40 e $60 \%$.

- Considerando uma saturação de 40 e $60 \%$, as doses de $\mathrm{P}$ que proporcionaram $90 \%$ da produção máxima, após 170 dias de crescimento, foram de 127 e $126 \mathrm{mg} / \mathrm{dm}^{3}$, respectivamente.

- Os níveis críticos de $\mathrm{P}$ no solo para $\mathrm{V}=40 \%$ e V $=60 \%$ foram, respectivamente, de 13,88 e 12,87 mg/dm ${ }^{3}$ e na parte aérea, de 0,14 e $0,12 \mathrm{dag} / \mathrm{kg}$.

\section{REFERÊNCIAS BIBLIOGRÁFICAS}

ALVAREZ V., V. H. Equilíbrio de formas disponíveis de fósforo e enxofre em dois Latossolos de Minas Gerais. 1974. 125f.. Dissertação (Mestrado em Solos e Nutrição de Plantas) - Universidade Federal de Viçosa, Viçosa, 1974.

BALIERO, F. C.; OLIVEIRA, I. G.; DIAS, L. E. Formação de mudas de Acacia holocericea: resposta à calagem, fósforo, potássio e enxofre. In: CONGRESSO BRASILEIRO DE CIÊNCIA DO SOLO, 25., 1995, Viçosa, MG. Resumos... Viçosa, MG: SBCS, 1995. p.830-832, 1158p.

BRAGA, J. M.; DEFELIPO, B. V. Determinação espectofotométrica de fósforo em extratos de solos e plantas. Revista Ceres, v.21, n.113, p.73-85, 1974.

R. Árvore, Viçosa-MG, v.28, n.6, p.785-792, 2004 
CARVALHO, P. E. R. Espécies florestais brasileiras: recomendações silviculturais, potencialidades e uso da madeira. Colombo: EMBRAPA - CNPF, 1994. 640p.

DANIEL, O. et al. Aplicação de fósforo em mudas de Acacia mangium Willd. Revista Árvore, v.21, n.2, p.163-168, 1997.

DIAS, L. E.; ALVAREZ V., V. H.; BRIENZA JR., S. Formação de mudas de Acacia mangium: 1. resposta a calcário e fósforo. In: CONGRESSO FLORESTAL BRASILEIRO, 6., 1990, Campos do Jordão. Anais... Campos do Jordão: SBS, SBEF, 1990. p.449-453, 801p.

DIAS, L. E. et al. Formação de mudas de taxibranco (Sclerolobium paniculatum Vog.). I. Resposta a calcário e fósforo. Pesquisa Agropecuária Brasileira, v.26, n.1, p.69-76, 1991.

DUBOC, E. et al. Fertilização de plântulas de Copaifera langsdorffii Desf. (óleo copaíba). Revista Cerne, v.2, n.2, p.31-47, 1996.

EMPRESA BRASILEIRA DE PESQUISA AGROPECUÁRIA - EMBRAPA. Centro Nacional de Pesquisa de Solos. 2. ed. Manual de métodos de análise de solo. Rio de Janeiro: CNPS, 1997. 212p.

FARIA, M. P. et al. Crescimento de leguminosas arbóreas em resposta a fósforo, nitrogênio, fungo micorrízico e rizóbio. II. Peltophorum dubium (Spreng.) Taub. Revista Árvore, v.19, n.4, p.433-446, 1995.

FERNANDES, L. A. et al. Crescimento inicial, níveis críticos de fósforo e frações fosfatadas em espécies florestais. Pesquisa Agropecuária Brasileira, v.35, n.6, p.1191-1198, 2000.

FURTINI NETO, A. E. Eficiência nutricional, cinética de absorção e frações fosfatadas em Eucalyptus spp. 1994. 99f..Tese (Doutorado em Solos e Nutrição de Plantas) - Universidade Federal de Viçosa, Viçosa, 1994.

FURTINI NETO, A. E. et al. Fertilização em reflorestamento com espécies nativas. In: GONÇALVES, J. L. M.; BENEDETTI, V. (Eds.). Nutrição e fertilização florestal. Piracicaba: IPEF, 2000. p.351-383.

R. Árvore, Viçosa-MG, v.28, n.6, p.785-792, 2004
GARCIA, N. C. P. Efeitos da calagem e de níveis de fósforo sobre o crescimento e composição mineral de mudas de cedro (Cedrela fissilis Vell.). 1986. 40f.. Dissertação (Mestrado em Solos e Nutrição de Plantas) - Universidade Federal de Viçosa, Viçosa, 1986.

GLASS, A. D. M. Plant nutrition. An introduction to current concepts. Boston: Jones and Bartlett Publishers, 1989. 234p.

LORENZI, H. Árvores brasileiras. Nova Odessa: Plantarum, 1992. 352p.

MALAVOLTA, E.; VITTI, G. C.; OLIVEIRA, S. A. Avaliação do estado nutricional das plantas: princípios e aplicações. 2. ed. Piracicaba: POTAFOS, 1997. 319p.

MEIRA NETO, J. A. A. Estudos florísticos, estruturais e ambientais nos estratos arbóreo e herbáceo-arbustivo de uma floresta estacional semidecidual em Viçosa, MG. 1997. 155f.. Tese (Doutorado em Biologia Vegetal) - Universidade Estadual de Campinas, Campinas, 1997.

MEIRA NETO, J. A. A. et al. Estrutura de uma floresta estacional semidecidual submontana em área diretamente afetada pela usina hidrelétrica de Pilar, Ponte Nova, Zona da Mata de Minas Gerais. Revista Árvore, v.21, n.3, p.337-344, 1997.

NOVAIS, R. F.; BARROS, N. F.; NEVES, J. C. L. Nutrição mineral do eucalipto. In: BARROS, N. F.; NOVAIS, R. F. (Eds.). Relação solo-eucalipto. Viçosa, MG: Folha de Viçosa, 1990. p.25-98.

NOVAIS, R. F.; SMYTH, T. J. Relação fonte-dreno de fósforo no solo. In: NOVAIS, R. F.; SMYTH, T. J. (Eds.). Fósforo em solo e planta em condições tropicais. Viçosa, MG: UFV, 1999. p.2-6.

PASSOS, M. A. A. Efeito da calagem e de fósforo no crescimento inicial da algaroba (Prosopis juliflora (SW) DC). 1994. 57f. Tese (Doutorado em Fitotecnia) Universidade Federal de Viçosa, Viçosa, 1994.

REIS, M. G. F. et al. Exigências nutricionais de mudas de Dalbergia nigra (Vell.) Fr. Allem

(Jacarandá-da-Bahia) produzidas em dois níveis de sombreamento. Revista Árvore, v.21, n.4, p.463-471, 1997.

SANTOS, E. Nossas madeiras. Belo Horizonte: Itatiaia, 1987. 313p. 